Baptist Health South Florida

Scholarly Commons @ Baptist Health South Florida

All Publications

$9-2020$

\title{
Case report of ablative magnetic resonance-guided stereotactic body radiation therapy for oligometastatic mesenteric lymph nodes from bladder cancer
}

\author{
Michael Chuong \\ Miami Cancer Institute, michaelchu@baptisthealth.net \\ Diane Alvarez \\ Miami Cancer Institute, dianeal@baptisthealth.net \\ Tino Romaguera \\ Miami Cancer Institute, AntinogenesR@baptisthealth.net \\ Kathryn Mittauer \\ Miami Cancer Institute, KathrynM@baptisthealth.net \\ Sonia Adamson \\ Miami Cancer Institute, SoniaAd@baptisthealth.net
}

See next page for additional authors

Follow this and additional works at: https://scholarlycommons.baptisthealth.net/se-all-publications

\section{Citation}

Therapeutic Radiology and Oncology (2020) 4:20

This Article -- Open Access is brought to you for free and open access by Scholarly Commons @ Baptist Health South Florida. It has been accepted for inclusion in All Publications by an authorized administrator of Scholarly Commons @ Baptist Health South Florida. For more information, please contact Carrief@baptisthealth.net. 


\section{Authors}

Michael Chuong, Diane Alvarez, Tino Romaguera, Kathryn Mittauer, Sonia Adamson, Alonso Gutierrez, Gustavo Luciani, Hayden Guerrero Olmo, and Antonio Ucar 


\title{
Case report of ablative magnetic resonance-guided stereotactic body radiation therapy for oligometastatic mesenteric lymph nodes from bladder cancer
}

\author{
Michael D. Chuong ${ }^{1}$, Diane Alvarez ${ }^{1}$, Tino Romaguera ${ }^{1}$, Kathryn E. Mittauer ${ }^{1}$, Sonia Adamson ${ }^{1}$, \\ Alonso N. Gutierrez ${ }^{1}$, Gustavo Luciani ${ }^{1}$, Hayden Guerrero ${ }^{1}$, Antonio Ucar ${ }^{2}$ \\ ${ }^{1}$ Department of Radiation Oncology, Miami Cancer Institute, Miami, FL, USA; ${ }^{2}$ Department of Medical Oncology, Miami Cancer Institute, Miami, \\ FL, USA \\ Correspondence to: Michael D. Chuong, MD. Miami Cancer Institute, Department of Radiation Oncology, 8900 North Kendall Drive, Miami, FL \\ 33176, USA. Email: michaelchu@baptisthealth.net.
}

\begin{abstract}
Several randomized trials have demonstrated that stereotactic body radiation therapy (SBRT) can significantly improve long-term clinical outcomes for patients with oligometastatic (OM) cancer, commonly defined as 1-5 metastatic lesions. Some lesions, especially those in the abdomen and pelvis, may not be appropriate candidates for receiving ablative dose if daily on-board computed tomography (CT) is used because of limited target lesion and normal anatomy visualization. Magnetic resonance imaging (MRI) inherently provides superior soft tissue delineation as compared to CT and only recently have MRguided linear accelerators (LINACs) become commercially available. MR-LINACs can also perform daily online adaptive replanning based on the current day's anatomy, further positioning this novel technology as a preferred means to safely deliver ablative dose, even to targets in anatomically challenging locations. Here we present the case of a 49-year-old man with bladder cancer who underwent cystectomy and developed metachronous disease in 2 mesenteric lymph nodes for which he received MR-guided SBRT with daily online adaptive replanning to a prescription dose of $50 \mathrm{~Gy}$ in 5 fractions. He achieved a significant radiographic response and did not experience significant treatment-related toxicity. We discuss unique advantages of MR guidance and novel applications, especially in the context of OM disease.
\end{abstract}

Keywords: Bladder cancer; magnetic resonance imaging (MRI); radiosurgery; radiotherapy

Received: 06 April 2020; Accepted: 04 June 2020; Published: 30 September 2020.

doi: $10.21037 /$ tro-20-37

View this article at: http://dx.doi.org/10.21037/tro-20-37

\section{Introduction}

Stereotactic body radiation therapy (SBRT) is established for the management of certain extracranial cancers, most notably those in the lung, in which ablative dose can safely be delivered and achieve excellent long-term local control (LC) (1). SBRT is not feasible for all extracranial tumors, however, because of suboptimal soft tissue visualization on pre-treatment computerized tomography $(\mathrm{CT})$ and the proximity of normal organs such as the bowel.

Daily image guidance using magnetic resonance (MR) has been available for several years at a limited although growing number of centers worldwide (2). MR-guided radiation therapy (MRgRT) offers several important advantages over CT-based image guidance that allows for safe tumor dose escalation while also better sparing normal organs, and thus may achieve a more favorable therapeutic ratio.

We present the following case in accordance with the CARE reporting checklist (available at http://dx.doi. org/10.21037/tro-20-37).

\section{Case report}

This is the case of a 49-year-old man with no significant 
past medical or surgical history who in January 2015 developed hematuria and underwent a workup including cystoscopy and CT scans that revealed a mass in the bladder with enlarged pelvic lymph nodes. Bladder biopsy was positive for invasive high-grade urothelial carcinoma. $\mathrm{He}$ underwent open radical cystectomy and prostatectomy with bilateral pelvic lymph node dissection and ileal neobladder urinary diversion in February 2015 for a $4.3 \mathrm{~cm}$, pT3bN2, invasive high-grade urothelial carcinoma of the bladder with 2 involved lymph nodes out of 12 . Surgical margins were negative.

Restaging CT scans several years later in May 2017 revealed a $3.7 \mathrm{~cm} \times 2.1 \mathrm{~cm}$ right adrenal mass inseparable from the right diaphragmatic crus that was consistent with distant metastasis. There was no evidence of disease recurrence at the primary site of disease in the pelvis or elsewhere.

He initiated treatment with cisplatin and gemcitabine in June 2017 for this metachronous OM lesion. CT scans in August 2017 showed that although the right adrenal mass was stable two lymph nodes in the central root of the mesentery were enlarging and suspicious for metastatic disease. After receiving 7 cycles of cisplatin and gemcitabine, CT scans in April 2018 showed that the right adrenal mass had increased to $3.4 \mathrm{~cm}$ and the mesenteric lymph nodes had increased to $3.1 \mathrm{~cm}$ and $1.6 \mathrm{~cm}$, respectively. There were no new lesions elsewhere. Cisplatin and gemcitabine were discontinued and he was started on atezolizumab.

He began the first cycle of atezolizumab in May 2018 and CT scans approximately 6 months later demonstrated complete resolution of the right adrenal metastasis while the mesenteric lymph nodes were stable. His disease remained well controlled for an additional year until there was again enlargement of the mesenteric lymph nodes to 5.9 and $1.6 \mathrm{~cm}$, respectively, after 22 cycles of atezolizumab. There were no additional sites of progression.

Because the mesenteric lymph nodes were the only site of metastatic disease for an extended period of time the multidisciplinary tumor board recommended ablative MRguided SBRT, which was delivered in late August 2019 on the ViewRay MRIdian Linac (ViewRay, Inc., Oakwood Village, $\mathrm{OH})$. The MRIdian Linac utilizes an onboard 0.35 T MR scanner and step-and-shoot radiation delivery. Simulation and pre-treatment MRI scans were obtained on the treatment machine using a True Fast Imaging with Steady State Free Precession (TRUFISP) sequence without contrast. An isotropic $3 \mathrm{~mm}$ setup margin was placed on the gross tumor volume (GTV) to define the planning target volume (PTV). Elective nodal regions were not treated given the small bowel surrounding the GTV. The GTV was $92.9 \mathrm{cc}$ while the PTV was $131.2 \mathrm{cc}$. Because treatment was delivered using a coached mid-inspiration breath hold technique, there was no internal target volume (ITV). The prescription dose was $50 \mathrm{~Gy}$ in 5 consecutive fractions; the biologically equivalent dose $\left(\mathrm{BED}_{10}\right)$ was $100 \mathrm{~Gy}$. An intentional hotspot of at least $120 \%$ of the prescription dose was delivered to as much of the GTV as possible while respecting normal organ constraints that included the following: small bowel (V35 $<0.5 \mathrm{cc} ; \mathrm{V} 40<0.03 \mathrm{cc}$ ), large bowel (V38<0.5 cc; V43<0.03 cc), combined kidneys (mean $<10 \mathrm{~Gy}$ ), liver (mean $<10 \mathrm{~Gy}$ ), cauda equina $(\mathrm{V} 20<0.5 \mathrm{cc})$. A $3 \mathrm{~mm}$ planning organ at risk (PRV) margin was uniformly expanded around the small bowel and used as an optimization structure, with the priority being to meet the normal organ constraints and the secondary goal being to maximize target volume coverage by the prescription dose. The prescription isodose line covered $72.6 \%$ of the PTV and $83.4 \%$ of the GTV. The mean and maximum dose to the GTV was 53.6 and $68.6 \mathrm{~Gy}$, respectively. The treatment plan used 13 inversely optimized beams with a total of 38 segments.

On each treatment day a volumetric MR TRUFISP scan was acquired in breath hold over 25 seconds (17 seconds is another option on the MRIdian Linac). Rigid registration of the target volumes and deformable registration of the normal organs was performed. The contours were then modified to reflect the current day's anatomy; the normal organs were recontoured within $3 \mathrm{~cm}$ from the periphery of the PTV. The predicted target volume and normal organ doses using the current day's anatomy and original treatment plan were evaluated. For each of the 5 fractions the predicted small bowel constraint was violated so the original plan (Figure 1A) was not used, but rather each fraction was reoptimized (Figure $1 B, C, D, E, F$ ) while the patient remained in treatment position and treatment was delivered using the new plan. This allowed all normal organ constraints to be satisfied while providing excellent dose coverage of the targeted lymph nodes. Continuous intrafraction motion was assessed in the sagittal plane throughout treatment delivery at a rate of 4 frames per second. Treatment was automatically paused if the at least $5 \%$ of the target position extended outside of a $3 \mathrm{~mm}$ threshold boundary; treatment resumed automatically when the target returned to within the defined threshold. The median time across all 5 fractions from the patient entering the treatment room to treatment delivery completion was 

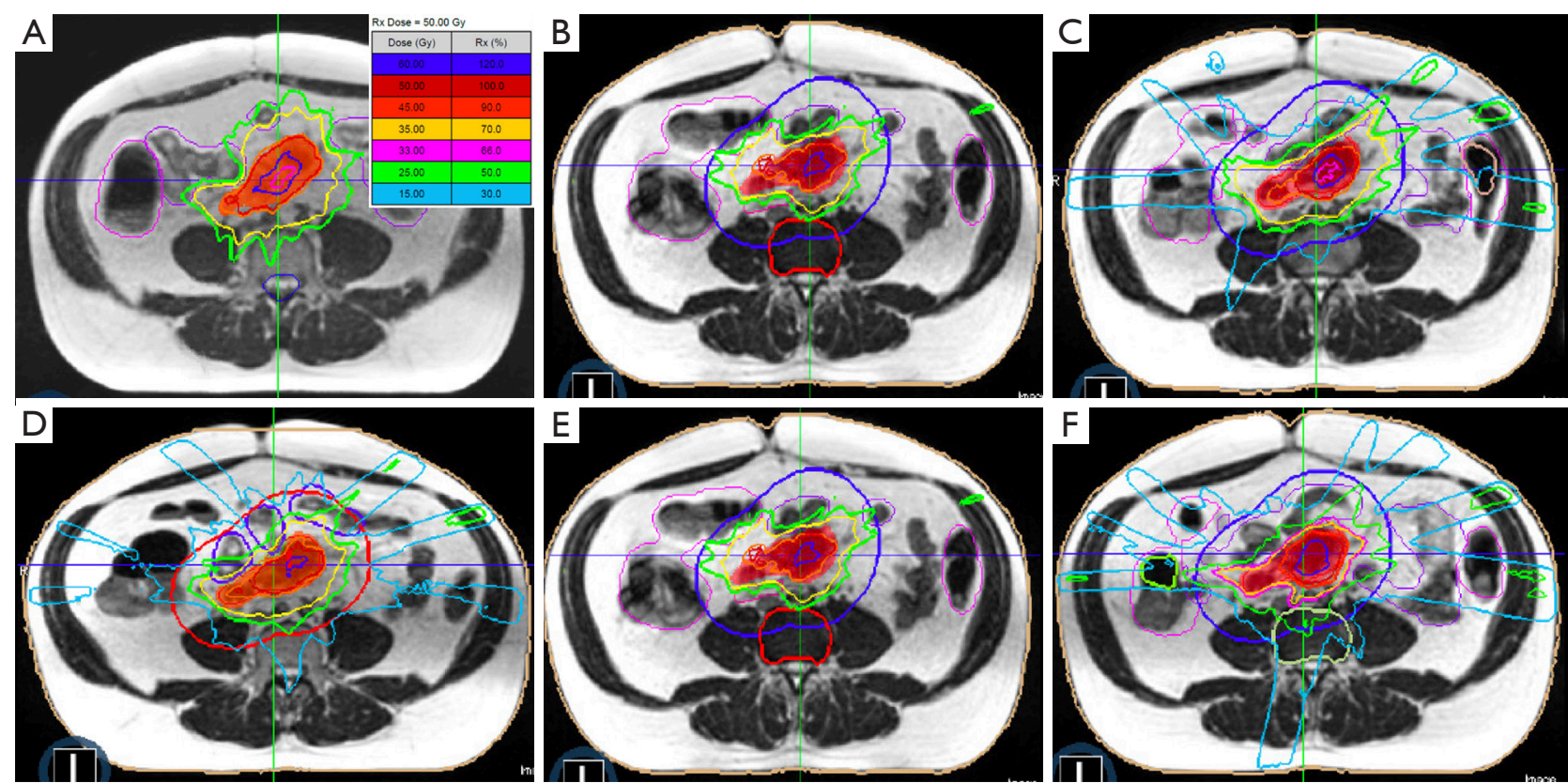

Figure 1 Isodose lines from the original treatment plan (A) compared to each daily fraction (B-F) that underwent online adaptive replanning due to a significant interfraction change in bowel positioning. The adapted plans ensured all normal organ dose constraints were met; target coverage varied depending on the current day's anatomy although most of the target received the prescribed dose for each fraction.

65 minutes, including time for initial positioning and online adaptive replanning. The median treatment delivery time in breath hold was 16 minutes.

The patient tolerated treatment very well and was able to complete MR-guided SBRT within 5 consecutive days as intended. He did not have severe toxicity and only experienced mild diarrhea and bloating.

CT scans in September 2019 demonstrated a reduction in the size of the treated mesenteric lesions to 2.3 and $1.2 \mathrm{~cm}$, respectively (Figure $2 A, B$ ). Two months later there was continued regression of the treated mesenteric lymph nodes to 1.8 and $1.2 \mathrm{~cm}$, respectively (Figure 2C).

He remained under close observation until a CT scan in March 2020 demonstrated progression of disease within the right adrenal gland and an upper abdominal mesenteric lymph node that was not previously treated with RT. The previously treated mesenteric lymph nodes continued to decrease in size, measuring 1.3 and $1 \mathrm{~cm}$, respectively. Because of disease progression the patient was started on weekly paclitaxel, with the potential for additional MRguided SBRT to be delivered to those sites pending review of treatment response on upcoming restaging scans. A timeline of his treatment course is summarized in Table 1.

Written informed consent was obtained from the patient for publication of this case report and any accompanying images. A copy of the written consent is available for review by the Editor-in-Chief of this journal.

\section{Discussion}

Radiation therapy (RT) for patients with distant metastasis has historically been limited to low dose palliation of symptoms such as pain or bleeding. However, dose escalated RT for OM disease (typically defined as 1-5 metastatic lesions) has received substantial attention after multiple randomized phase 2 trials demonstrated profound improvements in long-term progression free survival (PFS) and even potentially overall survival (OS) with the use of SBRT and chemotherapy versus chemotherapy alone (3-5). These trials included several different cancer types including breast, lung, and colorectal. Most recently, a phase 2 trial of OM prostate cancer patients showed that the SBRT arm achieved significantly improved PFS and enhanced systemic immune response compared to those randomized to observation (6). There may be a benefit of SBRT for other OM cancers including those of the bladder (7).

Despite increasing enthusiasm for SBRT in the management of OM disease, questions remain about ideal 

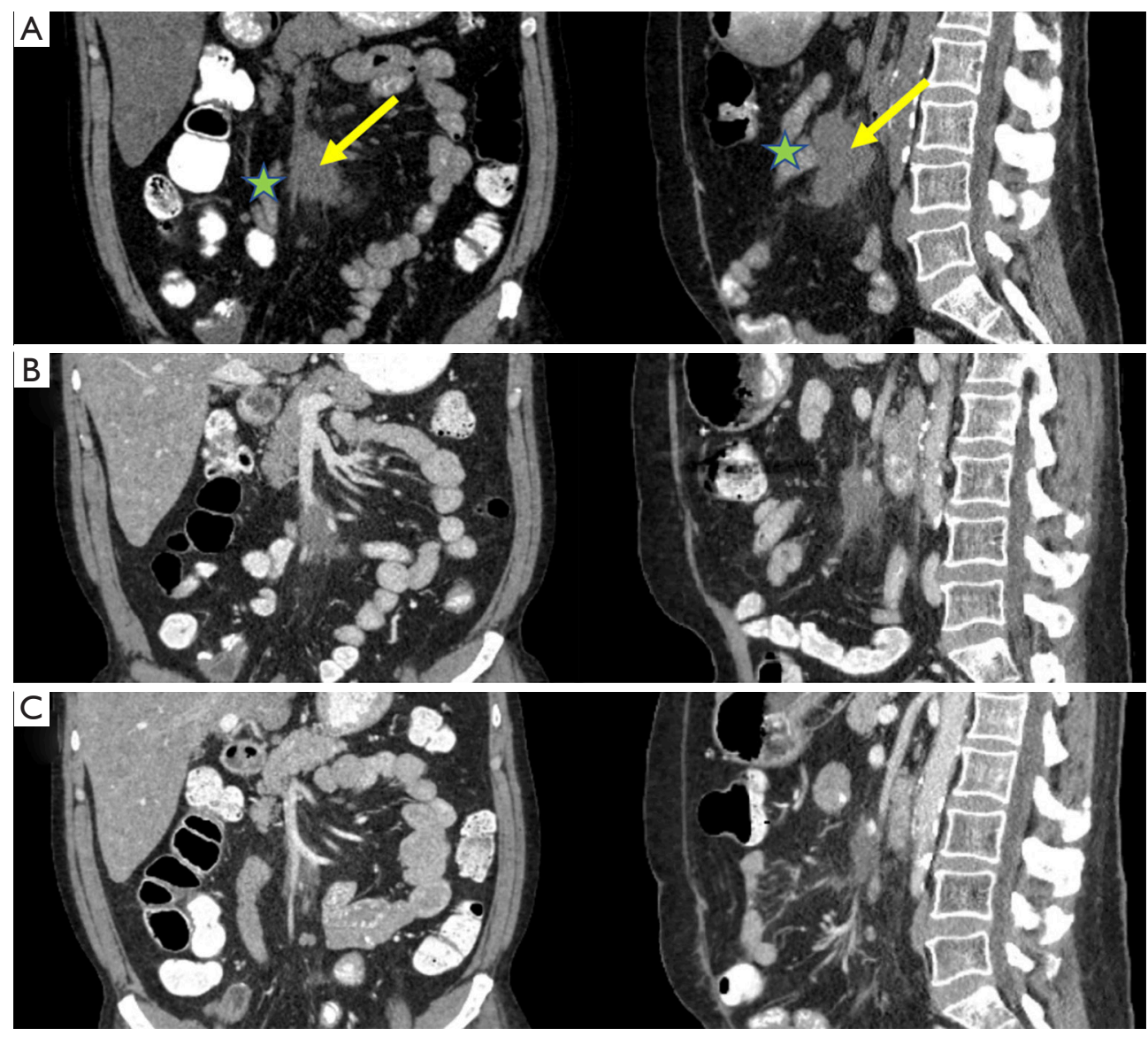

Figure 2 Oligometastatic mesenteric lymph nodes (arrow) that had progressed on systemic therapy prior to magnetic resonance-guided stereotactic body radiation therapy (A). Progressive radiographic response was observed 4 weeks (B) and 12 weeks (C) after ablation. The patient had no significant toxicity despite small bowel (star) abutting the target lesions.

patient selection and the optimal therapeutic approach (8). The aforementioned studies used a spectrum of radiation dose fractionation schedules, some being ablative (e.g., $\mathrm{BED}_{10} \geq 100 \mathrm{~Gy}$ ) while others were non-ablative, largely dependent on the anatomic location of the target lesion with respect to normal organs. While lesions such as those in the peripheral lung are excellent candidates to receive ablative dose in 5 or fewer fractions others such as abdominal LNs have historically not been because there is a considerable risk of severe toxicity due to the proximity of radiosensitive organs (e.g., bowel) (9). For example, Augugliaro et al. published a retrospective analysis of $13 \mathrm{OM}$ transitional cell bladder cancer patients who received RT predominantly to recurrent abdominal or pelvic LNs, most commonly prescribed to $25 \mathrm{~Gy}$ in 5 fractions $\left(\mathrm{BED}_{10}=37.5 \mathrm{~Gy}\right)(7)$. This non-ablative dose did not result in serious toxicity although restaging imaging after 3 months showed in-field progression among $38 \%$ of patients.

The emergence of MR-guided SBRT has begun to transform the field of radiation oncology (10). Not only does MRI offer superior soft tissue imaging over CT, thus obviating the need for fiducial marker placement, but MRguided radiation therapy devices also provide continuous intrafraction imaging of the tumor and surrounding normal organs throughout treatment allowing for smaller set up margins and ultimately target volumes. Additionally, while modifying the treatment plan to account for interfraction anatomic changes typically requires several days (when using CT guidance offline adaptation), MR-guided devices can create a new treatment plan within a few minutes based on the current day's anatomy (online adaptation) that facilitates ablative treatment. MR-guided SBRT has resulted in encouraging early clinical outcomes for OM disease and inoperable primary disease (11-14). Dose escalation using 
Table 1 Timeline from events since original diagnosis of bladder cancer

\begin{tabular}{|c|c|}
\hline Date & Event \\
\hline February 2015 & Radical cystectomy and prostatectomy, pelvic lymph node dissection (pT3bN2) \\
\hline May 2017 & Diagnosed with right adrenal gland metastasis \\
\hline June 2017 & Began gemcitabine/cisplatin \\
\hline May 2018 & Discontinued gemcitabine/cisplatin and started atezolizumab \\
\hline \multirow[t]{2}{*}{ November 2018} & Complete resolution of right adrenal metastasis \\
\hline & Stable mesenteric lymph nodes $(3.1 \mathrm{~cm}, 1.6 \mathrm{~cm})$ \\
\hline June 2019 & Enlarging mesenteric lymph nodes $(5.9 \mathrm{~cm}, 1.9 \mathrm{~cm})$ \\
\hline September 2019 & Decreasing size of mesenteric lymph nodes $(2.3 \mathrm{~cm}, 1.2 \mathrm{~cm})$ \\
\hline November 2019 & Decreasing size of mesenteric lymph nodes $(1.8 \mathrm{~cm}, 1.2 \mathrm{~cm})$ \\
\hline \multirow[t]{3}{*}{ March 2020} & Decreasing size of mesenteric lymph nodes $(1.3 \mathrm{~cm}, 1.0 \mathrm{~cm})$ \\
\hline & Progression in right adrenal gland and upper abdominal mesenteric lymph node \\
\hline & Began weekly paclitaxel \\
\hline
\end{tabular}

MR-guided SBRT is expected to improve outcomes far beyond what is achievable using CT guidance, which is especially relevant for patients with OM disease who may achieve long-term survival with aggressive management (15).

Several logistical considerations are pertinent for patients to be treated with an MR-LINAC. First, it must be verified that there is no physical contraindication for having an MRI scan due to implanted metallic objects or devices (i.e., prosthetic device, pacemaker). Second, patients must be able to tolerate lying in the bore of the MR-LINAC, which is more confined than a traditional LINAC. In our experience this has not been a major limitation for claustrophobic patients with the use of anxiolytic medication. Third, our MR-LINAC requires that a breath hold volumetric scan be performed for simulation and prior to each delivered fraction; this scan is ideally done over 25 seconds to achieve the highest image quality although can be done in 17 seconds if needed. We have not had a patient who was unable to tolerate at least the 17 second breath hold scan. Lastly, while we typically treat patients with thoracic or abdominal tumors in mid-inspiration breath hold to improve duty cycle efficiency, treatment in free breathing may be considered although the treatment time likely will increase.

Our patient had a remarkable response to MR-guided SBRT without having significant adverse effects. Despite his OM disease, his prognosis appears to be relatively favorable given that he was originally diagnosed with bladder cancer over 5 years ago, has not had widespread dissemination of his cancer, and now has achieved a remarkable response to SBRT. His case is notable because the delivery of ablative radiation therapy to mesenteric lymph nodes has not before been reported, to the best of our knowledge. Using MR guidance we were able to safely prescribe 50 Gy in 5 fractions $\left(\mathrm{BED}_{10}=100 \mathrm{~Gy}\right)$, which is significantly higher than what would typically be prescribed using CT guidance due to the tolerance of the small bowel (e.g., 25-35 Gy in 5 fractions; $\left.\mathrm{BED}_{10}=37.5-59.5 \mathrm{~Gy}\right)$. From the patient's perspective, receiving MR-guided SBRT was a positive experience since it was able to not only achieve tumor reduction despite progression on prior systemic therapies, but also offer him hope that he could still continue effectively fighting his disease. He is optimistic that his disease will remain controlled with chemotherapy and that he potentially might receive additional SBRT to any remaining sites of disease. 
The role of SBRT in the paradigm of OM management will almost certainly expand moving forward based on encouraging initial prospective data and the results of trials currently in progress that are expected to show a benefit for a wider array of cancer types. In that context, there will also likely be an increasing indication for daily MRI guidance as a means to optimize the therapeutic ratio of SBRT. Given that there is not yet extensive follow up using ablative dose with MR guidance, additional evaluation is needed to better understand long-term safety and efficacy from aggressive dosing regimens such as the one used for this patient. As such, a prospective trial is under development at our institution to evaluate outcomes of ablative MRguided SBRT delivered with online adaptive replanning for patients with $\mathrm{OM}$ disease.

\section{Acknowledgments}

Funding: None.

\section{Footnote}

Provenance and Peer Review: This article was commissioned by the Guest Editors (Chen-Hsi Hsieh, Hsin-Hua Nien, and Yu-Ming Huang) for the series "Precision Medicine for Cancer" published in Therapeutic Radiology and Oncology. The article was sent for external peer review organized by the Guest Editors and the editorial office.

Reporting Checklist: The authors have completed the CARE reporting checklist. Available at http://dx.doi.org/10.21037/ tro-20-37

Conflicts of Interest: All authors have completed the ICMJE uniform disclosure form (available at http://dx.doi. org/10.21037/tro-20-37). The series "Precision Medicine for Cancer" was commissioned by the editorial office without any funding or sponsorship. MDC reports grants, personal fees and non-financial support from ViewRay, personal fees and non-financial support from Sirtex, personal fees and non-financial support from Accuray, grants from AstraZeneca, outside the submitted work. KEM reports personal fees and non-financial support from ViewRay, other from MR Guidance, LLC, outside the submitted work. ANG reports personal fees and nonfinancial support from ViewRay, outside the submitted work. The authors have no other conflicts of interest to declare.
Ethical Statement: The authors are accountable for all aspects of the work in ensuring that questions related to the accuracy or integrity of any part of the work are appropriately investigated and resolved. All procedures performed in studies involving human participants were in accordance with the Declaration of Helsinki (as revised in 2013). This case report did not require institutional review board approval. Written informed consent was obtained from the patient for publication of this case report and any accompanying images. A copy of the written consent is available for review by the Editor-in-Chief of this journal.

Open Access Statement: This is an Open Access article distributed in accordance with the Creative Commons Attribution-NonCommercial-NoDerivs 4.0 International License (CC BY-NC-ND 4.0), which permits the noncommercial replication and distribution of the article with the strict proviso that no changes or edits are made and the original work is properly cited (including links to both the formal publication through the relevant DOI and the license). See: https://creativecommons.org/licenses/by-nc-nd/4.0/.

\section{References}

1. Videtic GM, Paulus R, Singh AK, et al. Long-term follow-up on nrg oncology rtog 0915 (ncctg n0927): A randomized phase 2 study comparing 2 stereotactic body radiation therapy schedules for medically inoperable patients with stage i peripheral non-small cell lung cancer. Int J Radiat Oncol Biol Phys 2019;103:1077-84.

2. Corradini S, Alongi F, Andratschke N, et al. Mr-guidance in clinical reality: Current treatment challenges and future perspectives. Radiat Oncol 2019;14:92.

3. Iyengar P, Wardak Z, Gerber DE, et al. Consolidative radiotherapy for limited metastatic non-small-cell lung cancer: A phase 2 randomized clinical trial. JAMA Oncol 2018;4:e173501.

4. Gomez DR, Tang C, Zhang J, et al. Local consolidative therapy vs. Maintenance therapy or observation for patients with oligometastatic non-small-cell lung cancer: Long-term results of a multi-institutional, phase ii, randomized study. J Clin Oncol 2019;37:1558-65.

5. Palma DA, Olson R, Harrow S, et al. Stereotactic ablative radiotherapy versus standard of care palliative treatment in patients with oligometastatic cancers (sabrcomet): A randomised, phase 2, open-label trial. Lancet 2019;393:2051-8.

6. Phillips R, Shi WY, Deek M, et al. Outcomes of 
observation vs stereotactic ablative radiation for oligometastatic prostate cancer: The oriole phase 2 randomized clinical trial. JAMA Oncol 2020;6:650-9.

7. Augugliaro M, Marvaso G, Ciardo D, et al. Recurrent oligometastatic transitional cell bladder carcinoma: Is there room for radiotherapy? Neoplasma 2019;66:160-5.

8. Zayed S, Correa RJM, Palma DA. Radiation in the treatment of oligometastatic and oligoprogressive disease: Rationale, recent data, and research questions. Cancer J 2020;26:156-65.

9. Jingu K, Matsuo Y, Onishi H, et al. Dose escalation improves outcome in stereotactic body radiotherapy for pulmonary oligometastases from colorectal cancer. Anticancer Res 2017;37:2709-13.

10. Hall WA, Paulson ES, van der Heide UA, et al. The transformation of radiation oncology using real-time magnetic resonance guidance: A review. Eur J Cancer 2019;122:42-52.

11. Rudra S, Jiang N, Rosenberg SA, et al. Using adaptive

doi: $10.21037 /$ tro-20-37

Cite this article as: Chuong MD, Alvarez D, Romaguera T, Mittauer KE, Adamson S, Gutierrez AN, Luciani G, Guerrero $\mathrm{H}$, Ucar A. Case report of magnetic resonance-guided stereotactic ablative radiation therapy for oligometastatic mesenteric lymph nodes from bladder cancer. Ther Radiol Oncol 2020;4:20. magnetic resonance image-guided radiation therapy for treatment of inoperable pancreatic cancer. Cancer Med 2019;8:2123-32.

12. Henke LE, Olsen JR, Contreras JA, et al. Stereotactic mr-guided online adaptive radiation therapy (smart) for ultracentral thorax malignancies: Results of a phase 1 trial. Adv Radiat Oncol 2018;4:201-9.

13. Tyran M, Cao M, Raldow AC, et al. Stereotactic magnetic resonance-guided online adaptive radiotherapy for oligometastatic breast cancer: A case report. Cureus 2018;10:e2368.

14. Henke L, Kashani R, Robinson C, et al. Phase i trial of stereotactic mr-guided online adaptive radiation therapy (smart) for the treatment of oligometastatic or unresectable primary malignancies of the abdomen. Radiother Oncol 2018;126:519-26.

15. Crane CH, O'Reilly EM. Ablative radiotherapy doses for locally advanced: Pancreatic cancer (lapc). Cancer J 2017;23:350-4. 